\title{
Human Blood-Vessel-Derived Stem Cells for Tissue Repair and Regeneration
}

\author{
Chien-Wen Chen, ${ }^{1,2,3}$ Mirko Corselli, ${ }^{4,5}$ Bruno Péault,, 5,6 and Johnny Huard ${ }^{2,3,7}$ \\ ${ }^{1}$ Department of Bioengineering, University of Pittsburgh, 236 Bridgeside Point II, 450 Technology Drive, PA 15219, USA \\ ${ }^{2}$ Department of Orthopedic Surgery, University of Pittsburgh Medical Center, Pittsburgh, PA 15219, USA \\ ${ }^{3}$ Stem Cell Research Center, University of Pittsburgh, Pittsburgh, PA 15219, USA \\ ${ }^{4}$ David Geffen School of Medicine, University of California at Los Angeles, Los Angeles, CA 90095, USA \\ ${ }^{5}$ Orthopaedic Hospital Research Center, University of California at Los Angeles, Los Angeles, CA 90095, USA \\ ${ }^{6}$ Center for Cardiovascular Science, Queen's Medical Research Institute, University of Edinburgh, Edinburgh EH16 4TJ, UK \\ ${ }^{7}$ McGowan Institute for Regenerative Medicine, University of Pittsburgh, Pittsburgh, PA 15219, USA
}

Correspondence should be addressed to Chien-Wen Chen, chc88@pitt.edu

Received 16 July 2011; Revised 31 October 2011; Accepted 1 November 2011

Academic Editor: Ken-ichi Isobe

Copyright ( $) 2012$ Chien-Wen Chen et al. This is an open access article distributed under the Creative Commons Attribution License, which permits unrestricted use, distribution, and reproduction in any medium, provided the original work is properly cited.

\begin{abstract}
Multipotent stem/progenitor cells with similar developmental potentials have been independently identified from diverse human tissue/organ cultures. The increasing recognition of the vascular/perivascular origin of mesenchymal precursors suggested blood vessels being a systemic source of adult stem/progenitor cells. Our group and other laboratories recently isolated multiple stem/progenitor cell subsets from blood vessels of adult human tissues. Each of the three structural layers of blood vessels: intima, media, and adventitia has been found to include at least one precursor population, that is, myogenic endothelial cells (MECs), pericytes, and adventitial cells (ACs), respectively. MECs and pericytes efficiently regenerate myofibers in injured and dystrophic skeletal muscles as well as improve cardiac function after myocardial infarction. The applications of ACs in vascular remodeling and angiogenesis/vasculogenesis have been examined. Our recent finding that MECs and pericytes can be purified from cryogenically banked human primary muscle cell culture further indicates their potential applications in personalized regenerative medicine.
\end{abstract}

\section{Introduction}

Multipotent adult stem/progenitor cells have been identified in nearly all human organs and extensively investigated to date [1-6]. For example, the human bone marrow (BM) functions as a diverse reservoir for several stem/progenitor cell populations, including hematopoietic stem cells (HSCs), multipotent mesenchymal stem/stromal cells (MSCs), and endothelial progenitor cells (EPCs) $[7,8]$. The human skeletal muscle contains committed myogenic precursors, skeletal myoblasts, primitive myogenic stem cells, and satellite cells [1]. On the other hand, the human fat harbors adipose progenitor cells and adipose-derived stem cells (ADSCs) which are functionally and phenotypically resembling the BM-MSCs $[9,10]$.
However, many of these stem/progenitor cell populations have been identified retrospectively in ex vivo tissue and organ cultures, such as multipotent adult progenitor cells (MAPCs), mesoangioblasts, and MSCs [10-13]. This obscures the origin and the native identity of these stem/progenitor cells in vivo. In order to fully utilize the developmental potentials and therapeutic potencies of the adult stem/progenitor cells, it is deemed important to understand whether these different populations of adult stem/progenitor cells are developmentally and/or hierarchically connected. Due to the phenotypical and functional similarities of several of these stem/progenitor cell populations, we hypothesized the existence of a common source in the human body. In this paper, we will discuss this newly emerged concept: blood-vessel-derived mesodermal 
stem/progenitor cells and their therapeutic applications in the futuristic personalized regenerative medicine.

\section{Stem/Progenitor Cell-Based Therapy}

The use of stem/progenitor cells for cell-based therapy is deemed promising owing to not only their high proliferative capacity and multilineage differentiation potential but also their functionality in secretion of trophic molecules and antienvironmental stress to promote cell survival. Specifically, adult stem/progenitor cells from an abundant autologous origin have the additional advantages over other stem cell types: high availability, no immunogenicity, low tumorigenicity, and with no associated ethical issues [14]. To efficiently repair/regenerate defective organs, the donor stem/progenitor cells are expected to possess desirable therapeutic properties, for example, minimal side effects, ability to integrate into host tissue, differentiation into desired cell lineages, paracrine effect, immunomodulation, regulation of tissue remodeling, and activation of endogenous repair/regeneration mechanisms [15-18].

The therapeutic potentials of adult stem/progenitor cells have been extensively investigated in the preclinical and clinical studies. For instance, MSCs have been widely explored in a number of phase I, II, and phase III clinical trials for several indications such as cardiovascular disease, stroke, multiple sclerosis/amyotrophic lateral sclerosis, Crohn's disease, and osteogenesis imperfecta [19-21]. Many other recent and ongoing human studies have chosen bone marrow mononuclear cells (BM-MNCs), skeletal myoblasts, EPCs, or ADSCs as their donor cell populations [16, 2224]. Unfortunately, in some cases, donor stem/progenitor cells initially demonstrated encouraging outcome but later on showed uneven success in clinical trials, especially in the treatment of cardiovascular disease $[16,23]$. Nevertheless, BM-MSCs, ADSCs, and other MSC-like mesodermal stem/progenitor cells still hold great promise for tissue repair and regeneration because of their multipotency, autologous availability, immune tolerance and regulation and robust paracrine secretion of trophic effectors.

\section{Blood Vessels as a Source of Stem/Progenitor Cells}

The evidence of the vascular wall as a source of stem/ progenitor cells has been demonstrated in the emerging hematopoietic system in the early development of human embryo and fetus [25]. The hematopoietic cells emerge in close vicinity to vascular endothelial cells in both intra and extraembryonic hematopoiesis. Specifically, a population of angiohematopoietic stem cells expressing flk-1 and angiotensin-converting enzyme (ACE) migrates from the para-aortic splanchnopleura into the ventral part of the aorta, where they give rise to hemogenic endothelial cells and subsequently hematopoietic cells [25]. Furthermore, hematopoietic cells also appear to develop from endothelium in the embryonic liver and fetal bone marrow, albeit at a much lower frequency.
The similarity between many human mesodermal stem/ progenitor cell populations that are retrospectively discovered in tissue/organ cultures has made us hypothesize that there exists a common, systemic source of stem/progenitor cells in the adult human body [26]. Blood vessels, which consist of three structural layers: tunica intima, tunica media, and tunica adventitia distribute throughout nearly all human organs where adult mesodermal stem/progenitor cells can be identified [27]. Apart from tunica intima, in which the subendothelial zone has been suggested as one of the sources of endothelial progenitor cells (EPCs), the possibility that other structural layers of the blood vessels harbor stem/progenitor cells was recently demonstrated in tunicae media and adventitia [26, 28, 29].

Perivascular cells, often known as vascular mural cells, are the cells that surround tunica intima of the blood vessels and constitute a major component of the vascular wall $[27,30]$. Microvascular pericytes, though similar but distinct from vascular smooth muscle cells, closely encircle endothelial cells in capillaries and microvessels (arterioles and venules) in most human tissues [30-32]. Pericytes are commonly regarded as a structural element of blood vessels that regulate vascular contractility and support the stability of blood vessels [33-35]. Intimate interactions between pericytes and endothelial cells tightly relate to the vascular growth, maturation, and remodeling [30, 34-36]. In addition, pericytes have been implicated in a number of pathological conditions, making them the potential targets for therapeutic interventions $[36,37]$.

Historically, the outmost layer of arteries and veins, the tunica adventitia, has been considered as a mere structural bystander constituted by collagen and fibroblasts. A number of recent findings have led to the reevaluation of the active role of tunica adventitia in cell trafficking, immune response mediation, and vascular remodeling [38]. The importance of the tunica adventitia in regenerative vascular medicine is highlighted by the numerous reports describing the presence of multipotent progenitors within the wall of arteries and veins $[29,39-42]$. In a vascular remodeling setting following an injury, it has been shown that adventitial cells (ACs) start a process of proliferation, migration, into the tunicae media and intima, and differentiation into smooth muscle cells $[41,43,44]$.

We have previously investigated whether the blood vessels contribute to stem/progenitor cell lineages other than hematopoietic cells. Through immunohistochemistry and flow cytometry analyses, we documented evidence showing the existence of rare subsets of human blood vessel derived stem cells (hBVSCs) in multiple human tissues, including skeletal muscle, fat, and placenta.

\section{The Three Musketeers: Myogenic Endothelial Cells, Pericytes, and Adventitial Cells}

We and other laboratories recently reported the existence of three distinct subpopulations of mesodermal precursors within the blood vessel walls through anatomic and molecular identifications. At least one precursor subset, that is, 

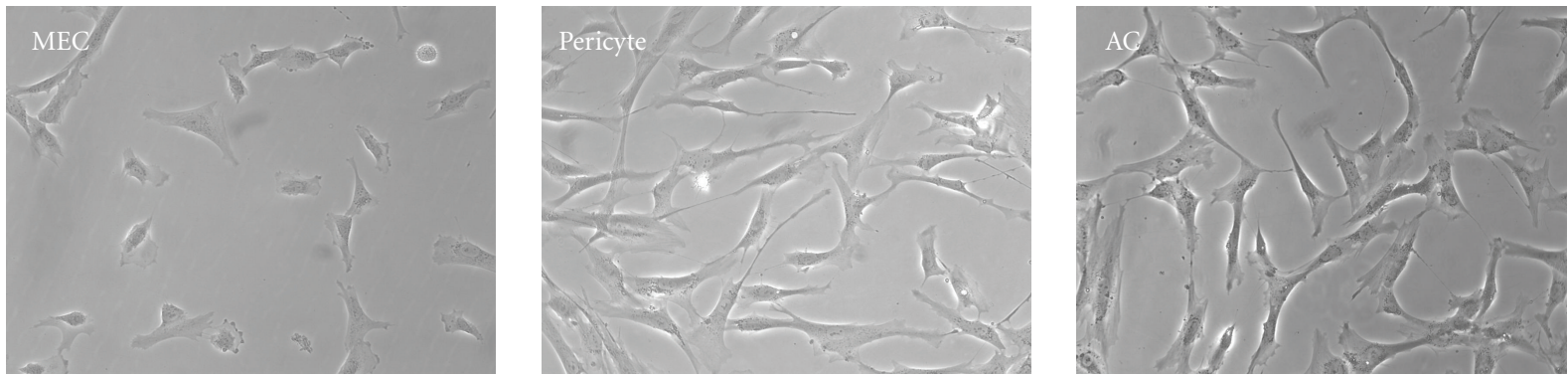

FIGURE 1: Distinct morphology of the three purified subpopulations of human blood-vessel-derived stem cells (hBVSCs) in culture: myogenic endothelial cell (MEC), pericyte, and adventitial cell (AC) (100x).

myogenic endothelial cells (MECs), pericytes, and adventitial cells (ACs) (Figure 1), was contained in each of the three tunicae of blood vessels: intima, media, and adventitia, respectively [26, 40, 45-47].

Myogenic endothelial cells (MECs), which suggest a developmental relationship between endothelial and myogenic cells, have been identified within the vasculatures of human skeletal muscle at a very low frequency $(<0.5 \%)$ [45]. MECs uniquely coexpressed myogenic (CD56) and endothelial cell markers (CD34 and CD144) and were identified by immunohistochemistry and flow cytometry. Using fluorescent-activated cell sorting (FACS), we were able to purify these cells to homogeny [45]. MECs (CD56+CD34+ CD144+CD45-) proliferate long term, retain a normal karyotype, survive better under oxidative stress than CD56+ myogenic cells, and are not tumorigenic [45]. Cultured MECs displayed mesenchymal developmental capacities, including myogenesis, osteogenesis, chondrogenesis, and adipogenesis, under appropriate inductive conditions in vitro [45]. Their stem cell characteristics were further confirmed by the expression of classic MSC markers and the mesodermal differentiations in culture from clonally derived MECs (Zheng et al., in revision). However, it is not clear yet whether MECs give rise to authentic MSCs in culture. Based on the phenotypic and functional similarities between MECs and the previously reported murine-muscle-derived stem cells (mMDSCs), we believe that MECs represent the human counterpart of mMDSCs. In addition to MECs, which are primarily located in the intimal compartment of the blood vessels within human skeletal muscle, other distinct subsets of multipotent stem/progenitor cells were recently found in the perivascular compartment of the vasculature (tunicae media and adventitia), not only within the skeletal muscle but throughout the human body [26, 40, 46, 47].

Though microvascular pericytes have long been considered to possess mesenchymal plasticity, the lack of a proper purification method undermined the characterization of this potential precursor population [48-50]. Recently, our group identified the native expression of classic MSC markers by microvascular pericytes and further discovered a collection of cell surface markers, that is, CD146+CD34-CD45-CD56- that enabled us to prospectively isolate homogenous pericyte populations by FACS from multiple human organs [26]. Purified pericytes prolif- erate long term and express CD146, NG2, PDGFR- $\beta$, alkaline phosphatase (ALP), and $\alpha$-smooth muscle actin ( $\alpha$-SMA), with the absence of endothelial cell markers, including von Willebrand factor (vWF), CD31, CD34, and CD144 [26, 46]. These cells can be efficiently expanded in vitro and demonstrate robust mesodermal developmental potentials, at the clonal level, by differentiating into osteogenic, chondrogenic, adipogenic, and myogenic lineages under suitable inductive conditions in vitro [26]. The MSC characteristics of these CD146+CD34-CD45-CD56- pericytes can be maintained for the long-term in culture. Their myogenic and osteogenic capacities were further displayed by transplantation into the muscle pocket of immunodeficient mice. To date, no tumorigenicity of pericytes has been reported $[26,46]$. We hypothesized that these cells are one of the developmental origins of MSCs [26].

In the past, fibroblasts that are capable of differentiating into myofibroblasts/smooth muscle cells (SMCs) following vascular injury have been regarded as the primary cellular component of the tunica adventitia $[38,51]$. Recent studies have gradually uncovered the true identity of the cells residing in this outmost layer of the blood vessels [42]. Cells located at the interface between the tunica adventitia and media, the so-called "vasculogenic zone", have been identified as CD34+CD31- and described as progenitors endowed with the ability to differentiate into endothelial cells and participate in the blood vessel formation as well as the pathogenesis of atherosclerosis [29, 41, 51]. The concept that the tunica adventitia functions as a reservoir for stem/progenitor cells is highlighted by a recent study in which a population of $\mathrm{CD} 34+\mathrm{CD} 31$ - progenitors residing in human saphenous vein was described [40]. These cells were localized in the tunica adventitia in situ and could be isolated and expanded at the clonal level in vitro. Our study also showed the stem cell characteristics of a CD34+CD31-CD146- nonpericyte perivascular cell population in the vasculature of human adipose and other tissues [47]. The FACS-purified CD34+CD31-CD146- population exhibited the phenotype and developmental potentials of MSCs. Furthermore, immunohistochemistry revealed that CD34+CD31-CD146- cells reside in tunica adventitia of blood vessels in multiple human organs and similar to pericytes, natively express classic MSC surface markers. 


\section{Myogenic Endothelial Cells for Skeletal Muscle Regeneration and Cardiac Repair}

The therapeutic potential of MECs was first tested in the severe combined immunodeficiency (SCID) mouse model of cardiotoxin-injured skeletal muscle [45]. MECs (CD56+CD34+CD144+CD45-) isolated from fresh human muscle biopsies were shown to regenerate skeletal myofibers more effectively than skeletal myoblasts (SkMs, CD56+/ CD34-/CD144-/CD45-), endothelial cells (ECs, CD34+/ CD144+/CD56-/CD45-), and unpurified primary muscle cells, with or without in vitro expansion. Injection of MECs into skeletal muscle of mdx/SCID mice, a disease model of Duchenne muscular dystrophy (DMD), also displayed an efficient regeneration of human skeletal myofibers, indicated by positive staining of both dystrophin and human lamin A/C [45]. Furthermore, after expansion in culture, clonal MECs exhibited robust chondrogenesis and osteogenesis in vivo after implantation into the hindlimb muscle pocket of SCID mice (Zheng et al., in revision). These results suggest the feasibility of utilizing MECs to treat various musculoskeletal disorders.

The application of MECs in the cardiovascular disease was examined in an immune-deficient mouse model of acute myocardial infarction (AMI) [52]. Myocardial infarction was induced in SCID-nonobese diabetic (NOD/SCID) mice by ligation of left anterior descending coronary artery (LAD), and cells were immediately injected into the ischemic myocardium. Cardiac function was assessed by echocardiography. The results demonstrated a significant improvement in cardiac contractility after intramyocardial injection of MECs when compared with injections of SkMs and ECs [52]. Transplanted MECs not only displayed robust engraftment within the infarcted myocardium but also stimulated angiogenesis, attenuated scar tissue formation, and promoted proliferation and survival of endogenous cardiomyocytes more effectively than the other two cell types [52]. This is presumably attributed to higher secretion of vascular endothelial growth factor (VEGF), a potent angiogenic factor, by MECs under hypoxia. Similar to mMDSCs, MECs regenerated significantly more fast-skeletal MHC-positive myofibers in the ischemic heart. A minor fraction of engrafted MECs differentiated into and/or fused with cardiomyocytes by expressing cardiomyocyte markers, cardiac troponin-T, and -I [52]. These findings suggest that MECs represent a promising stem cell subset within human skeletal muscle, an accessible autologous tissue source, for cardiac repair and regeneration.

\section{Pericytes for Tissue Repair and Regeneration}

The application of pericytes in regenerative medicine was first examined in SCID mouse models of injured and dystrophic skeletal muscle [26]. Microvascular pericytes freshly sorted by FACS from human skeletal muscle were injected into the hindlimb muscles of SCID-nonobese diabetic (NOD/SCID) mice that had been injured by intramuscular injection of cardiotoxin. The presence of regen- erating human spectrin-positive myofibers was detected by immunohistochemistry and confirmed by fluorescence in situ hybridization of central human nuclei [26]. Quantification showed that freshly sorted or long-term-cultured pericytes produced more human myofibers than purified SkMs and unpurified muscle cells, ruling out the possibility that the myogenic potential observed in pericytes resulted from a consequence of the contamination by myoblasts. Most importantly, this myogenic potential can be generalized to pericytes residing in nonmuscle tissues. Placenta-, white adipose tissue-, and pancreas-derived pericytes also exhibited high myogenic potential in culture and in vivo, yielding human dystrophin- or spectrin-positive myofibers upon transplanted into mdx/SCID or cardiotoxin-treated NOD/SCID mouse muscles [26]. Dellavalle et al. also showed that pericytes sorted from healthy and dystrophic human skeletal muscles by ALP expression regenerate human myofibers in muscles of dystrophic immunodeficient mice [46]. Very recently, the same group demonstrated that pericytes residing in the postnatal skeletal muscle natively participate in the skeletal myofiber development, and the satellite cell compartment and further contribute to the regeneration of injured/dystrophic skeletal muscle, using a transgenic label of inducible Alkaline Phosphatase CreERT2 [53]. These results indicate that pericytes play a role in muscle ontogeny and are endowed with robust myogenic potential that can be applied to skeletal muscle repair and regeneration.

With their inherent functions in the vascular physiology, pericytes seem to match the criteria of the ideal donor cell population for cardiovascular repair. Recently, we investigated the hypothesis that transplantation of cultured pericytes benefits the ischemic heart [54]. Briefly, long-termcultured human muscle pericytes were injected into acutely infarcted hearts of NOD/SCID mice. Echocardiography revealed a significant improvement of cardiac function in pericyte-injected hearts. Pericytes exhibited cardioprotective effects such as promotion of angiogenesis, reduction of scar formation, and inhibition of chronic inflammation (Chen et al., submitted). Moreover, a human pericyte-based smalldiameter vascular graft has been successfully engineered with high patency after long-term transplantation [55]. These data suggest that pericytes serve as a promising donor cell source for stem-cell-based cardiovascular therapy.

\section{Adventitial Cells Exhibit Multilineage Potential for Blood Vessel and Tissue Regeneration}

The potential application of ACs in the clinical settings has thus far been focused on the cardiovascular repair and regeneration. Campagnolo et al. recently demonstrated that CD34+CD31- ACs interact with endothelial cells and promote the formation and stabilization of the capillary-like structures [40]. Most importantly, injection of adventitial in a hindlimb ischemia mouse model cells showed a significant proangiogenic effect as demonstrated by a full blood flow recovery as early as 7 days after-injection, indicating the 
therapeutic potential of ACs in angiogenesis/vasculogenesis [40]. Very recently, Katare et al. further reported that transplantation of ACs improves the repair of infarcted hearts through angiogenesis involving microRNA-132 [56]. Together, these data indicate the therapeutic potential of ACs in ischemic tissue repair. Interestingly, the use of ACs derived from the umbilical artery, in conjunction with SMCs of the same source, in tissue-engineered small-caliber vessel constructs resulted in superior mechanical properties than the same constructs using cells derived from the umbilical vein, suggesting that ACs and SMCs originating from different sources may lead to distinct tissue characteristics in regenerative medicine [57].

The application of ACs is not limited to postnatal angiogenesis/vasculogenesis or cardiovascular repair. Though the myogenic potential of ACs remains to be determined, we and other authors have demonstrated that adventitial cells, regardless of their tissue of origin, display features typical of MSCs [40, 42, 47]. The ability of ACs to differentiate into major mesodermal cell lineages, including osteogenesis and chondrogenesis, suggests a likely contribution of these cells to the formation/regeneration of surrounding mesenchymal tissues after injury and further expands the potential clinical applications of this unique hBVSC subset into the field of musculoskeletal diseases [47]. Altogether, these reports suggest that rather than a passive constituent of the vascular wall, the adventitia is a dynamic reservoir of stem/progenitor cells that participate in vascular remodeling and regeneration of surrounding tissues.

\section{Cryopreserved Human Skeletal Muscle Culture as a Source of Myogenic hBVSCs}

Despite the successful isolations of various lineages of hBVSCs from fresh tissue biopsies, the prolonged procedure of FACS purification and subsequent culture expansion complicate the usage of these promising adult stem/progenitor cell populations. Specifically, this represents a major hurdle to the clinical translation of hBVSCs. We recently reported that long-term cryopreserved human primary skeletal muscle cell cultures (cryo-hPSMCs) include diverse cell populations; two subpopulations of hBVSCs, myogenic endothelial cells (MECs) and pericytes/perivascular stem cells (PSCs), can be purified from cryo-hPSMCs by FACS [58]. Due to the frequent loss of CD34 and CD144 expression in culture, another endothelial cell marker, UEA-1 receptor (UEA-1R), was used for purification in this case. CryoMECs express all three cell lineage markers (CD56+UEA$1 \mathrm{R}+\mathrm{CD} 146+\mathrm{CD} 45-)$, and cryo-PSCs express only CD146 (CD146+CD56-UEA-1R-CD45-). The preservation of the myogenic capacity of cryo-MECs and cryo-PSCs was demonstrated by the regeneration of human spectrin-positive myofibers after injections of newly sorted cells (with or without culture expansion) into the cardiotoxin-injured skeletal muscles of immunodeficient mice [58]. MECs showed the highest regenerative capacity in the injured mouse muscles, better than the unsorted muscle cells, CD56+ myoblasts, and cryo-PSCs. Cryo-PSCs remained myogenically superior to the unsorted cells and myoblasts. These findings suggest the feasibility to further purify subpopulations of the hBVSCs from banked human skeletal muscle cells, highlighting a new approach to extract therapeutic stem/progenitor cells from a cryogenically banked source for personalized regenerative medicine.

\section{Ontogeny and Heterogeneity, Innate Factors Affecting the Therapeutic Potency of Stem/Progenitor Cells?}

MSCs represent a highly heterogeneous population of widely studied but poorly defined multipotent stem/progenitor cells [59]. Indeed, Guilak et al. have shown that only $52 \%$ of the clones obtained from cultured ADSCs retain the ability to differentiate into two or more mesodermal cell lineages [60]. The presence of subsets of cells with limited or no differentiation potential within the conventional stromal cultures may therefore hamper the clinical efficacy of these promising stem/progenitor cells. This raised the possibility that clinical trials based on the transplantations of the total SVF or unfractionated MNCs often showed uneven success because of the variable frequency of progenitors within the total stroma [16, 23]. Additionally, mesenchymal progenitors have mostly been selected by their plastic adherence and expanded long-term in culture with reagents originated from animals, which ultimately limits their clinical use due to FDA regulations.

The identification of pericytes as, at least in part, the ancestors of MSCs has represented a breakthrough in the search for the true identity of MSCs [26]. Though this has raised the possibility that most MSCs, if not all, are derived from pericytes, other subsets of stem/progenitor cells residing in the blood vessel walls may constitute part of the MSC entity as described above [61]. The question of whether all MSCs originate from microvascular pericytes is partially answered by Tormin et al., who reported that, in bone marrow, MSCs can be derived not only from the subendothelial sinusoidal CD146+ cells, as previously demonstrated by Sacchetti et al., but also from the bonelining CD146 - cells $[62,63]$. Furthermore, using the genetic lineage tracing, Feng et al. recently reported MSCs from both pericyte and nonpericyte origins differentiate into odontoblasts and participate in tooth growth and repair in mice, suggesting that the pericyte contribution to MSCs may vary in different tissues and possibly depend on the density of the local vascularity [64].

Another good example that different subsets of stem/ progenitor cells contributing to the MSC entity is from the observation of the concurrent presence of two distinct subpopulations of perivascular multipotent progenitor cells, namely, CD34-CD31-CD146+ microvascular pericytes and CD34+CD31-CD146- adventitial cells, in the adipose tissue $[39,47]$. These two subpopulations of hBVSCs share in situ and in vitro expression of typical MSC surface markers, CD44, CD73, CD90, and CD105, but they are phenotypically and anatomically distinct $[39,47]$. Pericytes are indeed defined as CD45-CD34-CD31-CD146+ cells 
TABLE 1: Comparison of hBVSC subpopulations and bone marrow MSCs.

\begin{tabular}{|c|c|c|c|c|}
\hline Native location & $\begin{array}{c}\text { MEC } \\
\text { Intima }\end{array}$ & $\begin{array}{l}\text { Pericyte } \\
\text { Media }\end{array}$ & $\begin{array}{c}\text { AC } \\
\text { Adventitia }\end{array}$ & $\begin{array}{c}\text { BM-MSC } \\
\text { Bone marrow }\end{array}$ \\
\hline $\begin{array}{l}\text { Cell surface marker profile for } \\
\text { cell sorting }\end{array}$ & $\begin{array}{l}\text { CD34+ } \\
\text { CD45- } \\
\text { CD56+ } \\
\text { CD144+ }\end{array}$ & $\begin{array}{l}\text { CD34- } \\
\text { CD45- } \\
\text { CD56- } \\
\text { CD146+ }\end{array}$ & $\begin{array}{l}\text { CD31- } \\
\text { CD34+ } \\
\text { CD45- } \\
\text { CD146- }\end{array}$ & N/A \\
\hline $\begin{array}{l}\text { Classic MSC marker expression } \\
\text { in culture }\end{array}$ & $\begin{array}{l}\text { CD29+ } \\
\text { CD44+ } \\
\text { CD90+ } \\
\text { CD105+ }\end{array}$ & $\begin{array}{c}\text { CD44+ } \\
\text { CD73+ } \\
\text { CD90+ } \\
\text { CD105+ }\end{array}$ & $\begin{array}{l}\text { CD44+ } \\
\text { CD73+ } \\
\text { CD90+ } \\
\text { CD105+ }\end{array}$ & $\begin{array}{c}\text { CD29+ } \\
\text { CD44+ } \\
\text { CD73+ } \\
\text { CD90+ } \\
\text { CD105+ }\end{array}$ \\
\hline Differentiation in vitro & $\begin{array}{c}\text { Osteogenic }(+) \\
\text { Chondrogenic }(+) \\
\text { Adipogenic (ND) } \\
\text { Myogenic }(+)\end{array}$ & $\begin{array}{l}\text { Osteogenic }(+) \\
\text { Chondrogenic }(+) \\
\text { Adipogenic }(+) \\
\text { Myogenic }(+)\end{array}$ & $\begin{array}{c}\text { Osteogenic (+) } \\
\text { Chondrogenic }(+) \\
\text { Adipogenic (+) } \\
\text { Myogenic (ND) }\end{array}$ & $\begin{array}{c}\text { Osteogenic }(+) \\
\text { Chondrogenic }(+) \\
\text { Adipogenic }(+) \\
\text { Myogenic }(+)\end{array}$ \\
\hline Differentiation in vivo & Myogenesis & Myogenesis Osteogenesis & Vasculogenesis & $\begin{array}{c}\text { Osteogenesis } \\
\text { Chondrogenesis } \\
\text { Adipogenesis } \\
\text { Myogenesis } \\
\text { Cardiomyogenesis } \\
\text { Vasculogenesis }\end{array}$ \\
\hline Potential clinical application & $\begin{array}{l}\text { Skeletal musclere- } \\
\text { pair/regeneration; } \\
\text { Cardiac repair }\end{array}$ & $\begin{array}{c}\text { Skeletal muscle } \\
\text { repair/regeneration; } \\
\text { Vascular } \\
\text { repair/regeneration }\end{array}$ & $\begin{array}{c}\text { Vascular } \\
\text { repair/regeneration; } \\
\text { Cardiac repair }\end{array}$ & $\begin{array}{l}\text { Bone repair; } \\
\text { Cartilage repair; } \\
\text { Tendon/ligament } \\
\text { repair; skeletal } \\
\text { muscle repair; } \\
\text { Vascular repair; } \\
\text { Cardiac repair; } \\
\text { Wound healing; } \\
\text { Immunoregulation }\end{array}$ \\
\hline
\end{tabular}

MEC: myogenic endothelial cell; AC: adventitial cell; BM-MSC: bone marrow mesenchymal stem/stromal cells; N/A: not available; ND: not determined.

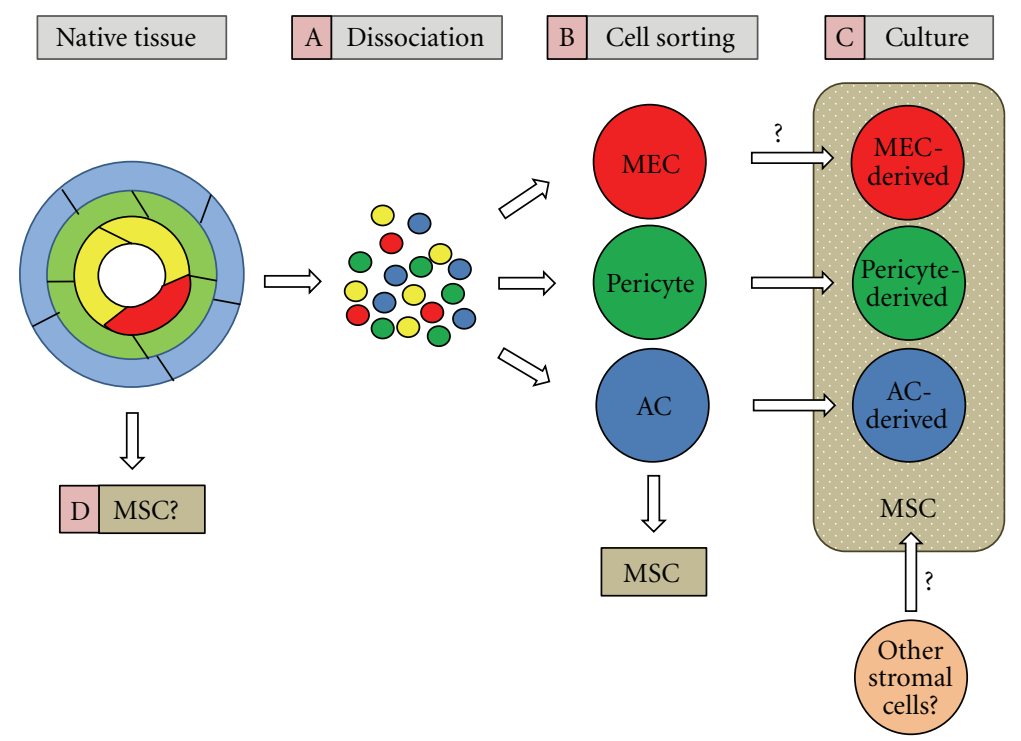

FIGURE 2: Schematic depiction of hBVSCs at the origin of mesenchymal stem/stromal cells (MSCs). (A) hBVSCs, including myogenic endothelial cells (MECs, red), pericytes (green), and adventitial cells (AC, blue), are dissociated from fresh muscle biopsy and separated from endothelial cells (yellow) and other cell types. (B) Dissociated cells are purified to homogeneity by fluorescence-activated cell sorting (FACS) and newly sorted MECs, pericytes, and ACs already exhibit multilineage developmental potentials. (C) FACS-purified pericytes, ACs, and possibly MECs give rise to authentic MSCs in long-term culture. (D) Nevertheless, whether native hBVSCs serve as a source of MSCs in situ and participate in tissue repair and regeneration remains an open question. 
tightly surrounding microvessels, whereas CD45-CD34+ CD31-CD146- adventitial cells are located in the outmost layer of larger blood vessels in the human adipose, a literally unlimited tissue source. Both subpopulations of cells are endowed with multilineage mesenchymal differentiation capacity at the clonal level and represent ideal candidates for the treatment of musculoskeletal and vascular diseases [39, 40, 65]. Above all, both subpopulations are abundant in lipoaspirate, and with the definitive phenotypes of each hBVSC fraction, we can readily enrich both fractions by cell sorting in order to improve their therapeutic efficiency and safety [47]. Table 1 summarizes the basic characteristics of hBVSC subpopulations and BM-MSCs as well as their potential clinical applications.

Nevertheless, while all, or at least part of, the three hBVSC subpopulations contributing to the MSC entity in culture are gradually becoming an accepted notion; whether the multilineage potentials are natively present within hBVSC subsets and subsequently responsive to pathological stimulations in vivo remains to be investigated (Figure 2). Ultimately, the current therapeutic strategy based on the transplantation of unfractionated stromal cells may in the near future be replaced by the purification, combination, and direct reinfusion of the distinct subsets of hBVSCs, devoid of cells with none or a restricted regenerative potential.

\section{Conclusion}

In this paper, we discussed the newly emerged concept of blood vessels as a systemic source of adult stem/progenitor cells. Three subpopulations of hBVSCs, that is, MECs, pericytes, and ACs have been respectively isolated from different layers of the blood vessels and examined in detail for their developmental capacities and therapeutic potentials in tissue repair and regeneration. Besides, the fresh tissue biopsy, the cryogenically banked primary human skeletal muscle culture was shown to be an alternative source of myogenic subsets of hBVSCs, shedding new lights on the future of the personalized regenerative medicine. Finally, the identification of the precise phenotypes of distinct hBVSC subpopulations represents not only an important milestone for understanding the nature and origin of MSCs but also a crucial step toward the improvement of stem/progenitor cellbased therapies.

\section{References}

[1] B. Peault, M. Rudnicki, Y. Torrente et al., "Stem and progenitor cells in skeletal muscle development, maintenance, and therapy," Molecular Therapy, vol. 15, no. 5, pp. 867-877, 2007.

[2] P. A. Zuk, M. Zhu, P. Ashjian et al., "Human adipose tissue is a source of multipotent stem cells," Molecular Biology of the Cell, vol. 13, no. 12, pp. 4279-4295, 2002.

[3] J. G. Toma, M. Akhavan, K. J. L. Fernandes et al., "Isolation of multipotent adult stem cells from the dermis of mammalian skin," Nature Cell Biology, vol. 3, no. 9, pp. 778-784, 2001.

[4] Y. Choi, M. Ta, F. Atouf, and N. Lumelsky, "Adult pancreas generates multipotent stem cells and pancreatic and nonpan- creatic progeny," Stem Cells, vol. 22, no. 6, pp. 1070-1084, 2004.

[5] E. Messina, L. De Angelis, G. Frati et al., "Isolation and expansion of adult cardiac stem cells from human and murine heart," Circulation Research, vol. 95, no. 9, pp. 911-921, 2004.

[6] Y.-C. Hsu, D.-C. Lee, and I.-M. Chiu, "Neural stem cells, neural progenitors, and neurotrophic factors," Cell Transplantation, vol. 16, no. 2, pp. 133-150, 2007.

[7] C. B. Ballas, S. P. Zielske, and S. L. Gerson, "Adult bone marrow stem cells for cell and gene therapies: implications for greater use," Journal of Cellular Biochemistry, vol. 38, pp. 20$28,2002$.

[8] H. Chao and K. K. Hirschi, "Hemato-vascular origins of endothelial progenitor cells?” Microvascular Research, vol. 79, no. 3, pp. 169-173, 2010.

[9] W. Tang, D. Zeve, J. M. Suh et al., "White fat progenitor cells reside in the adipose vasculature," Science, vol. 322, no. 5901, pp. 583-586, 2008.

[10] J. M. Gimble, A. J. Katz, and B. A. Bunnell, "Adipose-derived stem cells for regenerative medicine," Circulation Research, vol. 100, no. 9, pp. 1249-1260, 2007.

[11] Y. Jiang, B. N. Jahagirdar, R. L. Reinhardt et al., "Pluripotency of mesenchymal stem cells derived from adult marrow," Nature, vol. 418, no. 6893, pp. 41-49, 2002.

[12] M. G. Minasi, M. Riminucci, L. De Angelis et al., "The mesoangioblast: a multipotent, self-renewing cell that originates from the dorsal aorta and differentiates into most mesodermal tissues," Development, vol. 129, no. 11, pp. 2773-2783, 2002.

[13] F. P. Barry and J. M. Murphy, "Mesenchymal stem cells: clinical applications and biological characterization," International Journal of Biochemistry and Cell Biology, vol. 36, no. 4, pp. 568584, 2004.

[14] A. R. Akar, S. Durdu, T. Corapcioglu, and U. Ozyurda, "Regenerative medicine for cardiovascular disorders-new milestones: adult stem cells," Artificial Organs, vol. 30, no. 4, pp. 213-232, 2006.

[15] V. F. M. Segers and R. T. Lee, "Stem-cell therapy for cardiac disease," Nature, vol. 451, no. 7181, pp. 937-942, 2008.

[16] S. J. Joggerst and A. K. Hatzopoulos, "Stem cell therapy for cardiac repair: benefits and barriers," Expert Reviews in Molecular Medicine, vol. 11, article e20, 2009.

[17] C.-W. Chen, E. Montelatici, M. Crisan et al., "Perivascular multi-lineage progenitor cells in human organs: regenerative units, cytokine sources or both?" Cytokine and Growth Factor Reviews, vol. 20, no. 5-6, pp. 429-434, 2009.

[18] K. R. Chien, I. J. Domian, and K. K. Parker, "Cardiogenesis and the complex biology of regenerative cardiovascular medicine," Science, vol. 322, no. 5907, pp. 1494-1497, 2008.

[19] H. K. Salem and C. Thiemermann, "Mesenchymal stromal cells: current understanding and clinical status," Stem Cells, vol. 28, no. 3, pp. 585-596, 2010.

[20] D. Karussis, C. Karageorgiou, A. Vaknin-Dembinsky et al., "Safety and immunological effects of mesenchymal stem cell transplantation in patients with multiple sclerosis and amyotrophic lateral sclerosis," Archives of Neurology, vol. 67, no. 10, pp. 1187-1194, 2010.

[21] F. Locatelli, A. Bersano, E. Ballabio et al., "Stem cell therapy in stroke," Cellular and Molecular Life Sciences, vol. 66, no. 5, pp. 757-772, 2009.

[22] H. C. Ott, B. H. Davis, and D. A. Taylor, "Cell therapy for heart failure -muscle, bone marrow, blood, and cardiac-derived stem cells," Seminars in Thoracic and Cardiovascular Surgery, vol. 17, no. 4, pp. 348-360, 2005. 
[23] M. F. Piepoli, "Transplantation of progenitor cells and regeneration of damaged myocardium: more facts or doubts? Insights from experimental and clinical studies," Journal of Cardiovascular Medicine, vol. 10, no. 8, pp. 624-634, 2009.

[24] D. P. Sieveking and M. K. Ng, "Cell therapies for therapeutic angiogenesis: back to the bench," Vascular Medicine, vol. 14, no. 2, pp. 153-166, 2009.

[25] M. Tavian, B. Zheng, E. Oberlin et al., "The vascular wall as a source of stem cells," Annals of the New York Academy of Sciences, vol. 1044, no. 1, pp. 41-50, 2005.

[26] M. Crisan, S. Yap, L. Casteilla et al., "A perivascular origin for mesenchymal stem cells in multiple human organs," Cell Stem Cell, vol. 3, no. 3, pp. 301-313, 2008.

[27] V. Kumar, N. Fausto, and A. Abbas, Robbins \& Cotran Pathologic Basis of Disease, Blood Vessels, chapter 11, Saunders, 7th edition, 2004.

[28] D. Tilki, H. P. Hohn, B. Ergün, S. Rafii, and S. Ergün, "Emerging biology of vascular wall progenitor cells in health and disease," Trends in Molecular Medicine, vol. 15, no. 11, pp. 501-509, 2009.

[29] E. Zengin, F. Chalajour, U. M. Gehling et al., "Vascular wall resident progenitor cells: a source for postnatal vasculogenesis," Development, vol. 133, no. 8, pp. 1543-1551, 2006.

[30] A. Armulik, A. Abramsson, and C. Betsholtz, "Endothelial/pericyte interactions," Circulation Research, vol. 97, no. 6, pp. 512-523, 2005.

[31] D. J. Crocker, T. M. Murad, and J. C. Geer, "Role of the pericyte in wound healing. An ultrastructural study," Experimental and Molecular Pathology, vol. 13, no. 1, pp. 51-65, 1970.

[32] E. R. Andreeva, I. M. Pugach, D. Gordon, and A. N. Orekhov, "Continuous subendothelial network formed by pericyte-like cells in human vascular bed," Tissue and Cell, vol. 30, no. 1, pp. 127-135, 1998.

[33] D. von Tell, A. Armulik, and C. Betsholtz, "Pericytes and vascular stability," Experimental Cell Research, vol. 312, no. 5, pp. 623-629, 2006.

[34] H. K. Rucker, H. J. Wynder, and W. E. Thomas, "Cellular mechanisms of CNS pericytes," Brain Research Bulletin, vol. 51, no. 5, pp. 363-369, 2000.

[35] P. Dore-Duffy and J. C. La Manna, "Physiologic angiodynamics in the brain," Antioxidants and Redox Signaling, vol. 9, no. 9, pp. 1363-1371, 2007.

[36] F. Kuhnert, B. Y. Y. Tam, B. Sennino et al., "Soluble receptormediated selective inhibition of VEGFR and PDGFR $\beta$ signaling during physiologic and tumor angiogenesis," Proceedings of the National Academy of Sciences of the United States of America, vol. 105, no. 29, pp. 10185-10190, 2008.

[37] P. Lindahl, B. R. Johansson, P. Leveen, and C. Betsholtz, "Pericyte loss and microaneurysm formation in PDGF-Bdeficient mice," Science, vol. 277, no. 5323, pp. 242-245, 1997.

[38] M. W. Majesky, X. R. Dong, V. Hoglund, W. M. Mahoney Jr., and G. Daum, "The adventitia: a dynamic interface containing resident progenitor cells," Arteriosclerosis, Thrombosis, and Vascular Biology, vol. 31, no. 7, pp. 1530-1539, 2011.

[39] L. Zimmerlin, V. S. Donnenberg, M. E. Pfeifer et al., "Stromal vascular progenitors in adult human adipose tissue," Cytometry Part A, vol. 77, no. 1, pp. 22-30, 2010.

[40] P. Campagnolo, D. Cesselli, A. Al Haj Zen et al., "Human adult vena saphena contains perivascular progenitor cells endowed with clonogenic and proangiogenic potential," Circulation, vol. 121, no. 15, pp. 1735-1745, 2010.

[41] Y. Hu, Z. Zhang, E. Torsney et al., "Abundant progenitor cells in the adventitia contribute to atheroscleroses of vein grafts in
ApoE-deficient mice," The Journal of Clinical Investigation, vol. 113, no. 9, pp. 1258-1265, 2004.

[42] A. Hoshino, H. Chiba, K. Nagai, G. Ishii, and A. Ochiai, "Human vascular adventitial fibroblasts contain mesenchymal stem/progenitor cells," Biochemical and Biophysical Research Communications, vol. 368, no. 2, pp. 305-310, 2008.

[43] Y. Shi, J. E. O’Brien, A. Fard, J. D. Mannion, D. Wang, and A. Zalewski, "Adventitial myofibroblasts contribute to neointimal formation in injured porcine coronary arteries," Circulation, vol. 94, no. 7, pp. 1655-1664, 1996.

[44] S. Oparil, S. J. Chen, Y. F. Chen, J. N. Durand, L. Allen, and J. A. Thompson, "Estrogen attenuates the adventitial contribution to neointima formation in injured rat carotid arteries," Cardiovascular Research, vol. 44, no. 3, pp. 608-614, 1999.

[45] B. Zheng, B. Cao, M. Crisan et al., "Prospective identification of myogenic endothelial cells in human skeletal muscle," Nature Biotechnology, vol. 25, no. 9, pp. 1025-1034, 2007.

[46] A. Dellavalle, M. Sampaolesi, R. Tonlorenzi et al., "Pericytes of human skeletal muscle are myogenic precursors distinct from satellite cells," Nature Cell Biology, vol. 9, no. 3, pp. 255-267, 2007.

[47] M. Corselli, C. W. Chen, and B. Sun, "The tunica adventitia of human arteries and veins as a source of mesenchymal stem cells," Stem Cells and Development. In press.

[48] C. T. Brighton, D. G. Lorich, R. Kupcha, T. M. Reilly, A. R. Jones, and R. A. Woodbury, "The pericyte as a possible osteoblast progenitor cell," Clinical Orthopaedics and Related Research, no. 275, pp. 287-299, 1992.

[49] M. J. Doherty, B. A. Ashton, S. Walsh, J. N. Beresford, M. E. Grant, and A. E. Canfield, "Vascular pericytes express osteogenic potential in vitro and in vivo," Journal of Bone and Mineral Research, vol. 13, no. 5, pp. 828-838, 1998.

[50] C. Farrington-Rock, N. J. Crofts, M. J. Doherty, B. A. Ashton, C. Griffin-Jones, and A. E. Canfield, "Chondrogenic and adipogenic potential of microvascular pericytes," Circulation, vol. 110, no. 15, pp. 2226-2232, 2004.

[51] Y. $\mathrm{Hu}$ and Q. Xu, "Adventitial biology: differentiation and function," Arteriosclerosis, Thrombosis, and Vascular Biology, vol. 31, no. 7, pp. 1523-1529, 2011.

[52] M. Okada, T. R. Payne, B. Zheng et al., "Myogenic endothelial cells purified from human skeletal muscle improve cardiac function after transplantation into infarcted myocardium," Journal of the American College of Cardiology, vol. 52, no. 23, pp. 1869-1880, 2008.

[53] A. Dellavalle, G. Maroli, D. Covarello et al., "Pericytes resident in postnatal skeletal muscle differentiate into muscle fibres and generate satellite cells," Nature Communications, vol. 2, no. 1, Article ID 499, 2011.

[54] C.-W. Chen, K. Tobita, B. Peault et al., "Purified human muscle-derived pericytes support formation of vascular structures and promote angiogenesis after myocardial infarction," Circulation, vol. 120, p. S1053, 2009.

[55] W. He, A. Nieponice, L. Soletti et al., "Pericyte-based human tissue engineered vascular grafts," Biomaterials, vol. 31, no. 32, pp. 8235-8244, 2010.

[56] R. Katare, F. Riu, K. Mitchell et al., "Transplantation of human pericyte progenitor cells improves the repair of infarcted heart through activation of an angiogenic program involving microRNA-132/novelty and significance," Circulation Research, vol. 109, no. 8, pp. 894-906, 2011.

[57] R. Gauvin, M. Guillemette, T. Galbraith et al., "Mechanical properties of tissue-engineered vascular constructs produced 
using arterial or venous cells," Tissue Engineering A, vol. 17, no. 15-16, pp. 2049-2059, 2011.

[58] B. Zheng, "Isolation of myogenic stem cells from cultures of cryopreserved human skeletal muscle," Cell Transplantation. In press.

[59] M. Pevsner-Fischer, S. Levin, and D. Zipori, "The origins of mesenchymal stromal cell heterogeneity," Stem Cell Reviews and Reports, pp. 1-9, 2011.

[60] F. Guilak, K. E. Lott, H. A. Awad et al., "Clonal analysis of the differentiation potential of human adipose-derived adult stem cells," Journal of Cellular Physiology, vol. 206, no. 1, pp. 229237, 2006.

[61] A. I. Caplan, "All MSCs are pericytes?" Cell Stem Cell, vol. 3, no. 3, pp. 229-230, 2008.

[62] B. Sacchetti, A. Funari, S. Michienzi et al., "Self-renewing osteoprogenitors in bone marrow sinusoids can organize a hematopoietic microenvironment," Cell, vol. 131, no. 2, pp. 324-336, 2007.

[63] A. Tormin, O. Li, J. C. Brune et al., "CD146 expression on primary nonhematopoietic bone marrow stem cells is correlated with in situ localization," Blood, vol. 117, no. 19, pp. 5067-5077, 2011.

[64] J. Feng, A. Mantesso, C. De Bari, A. Nishiyama, and P. T. Sharp, "Dual origin of mesenchymal stem cells contributing to organ growth and repair," Proceedings of the National Academy of Sciences of the United States of America, vol. 108, no. 16, pp. 6503-6508, 2011.

[65] F. S. Tedesco, A. Dellavalle, J. Diaz-Manera, G. Messina, and G. Cossu, "Repairing skeletal muscle: regenerative potential of skeletal muscle stem cells," The Journal of Clinical Investigation, vol. 120, no. 1, pp. 11-19, 2010. 

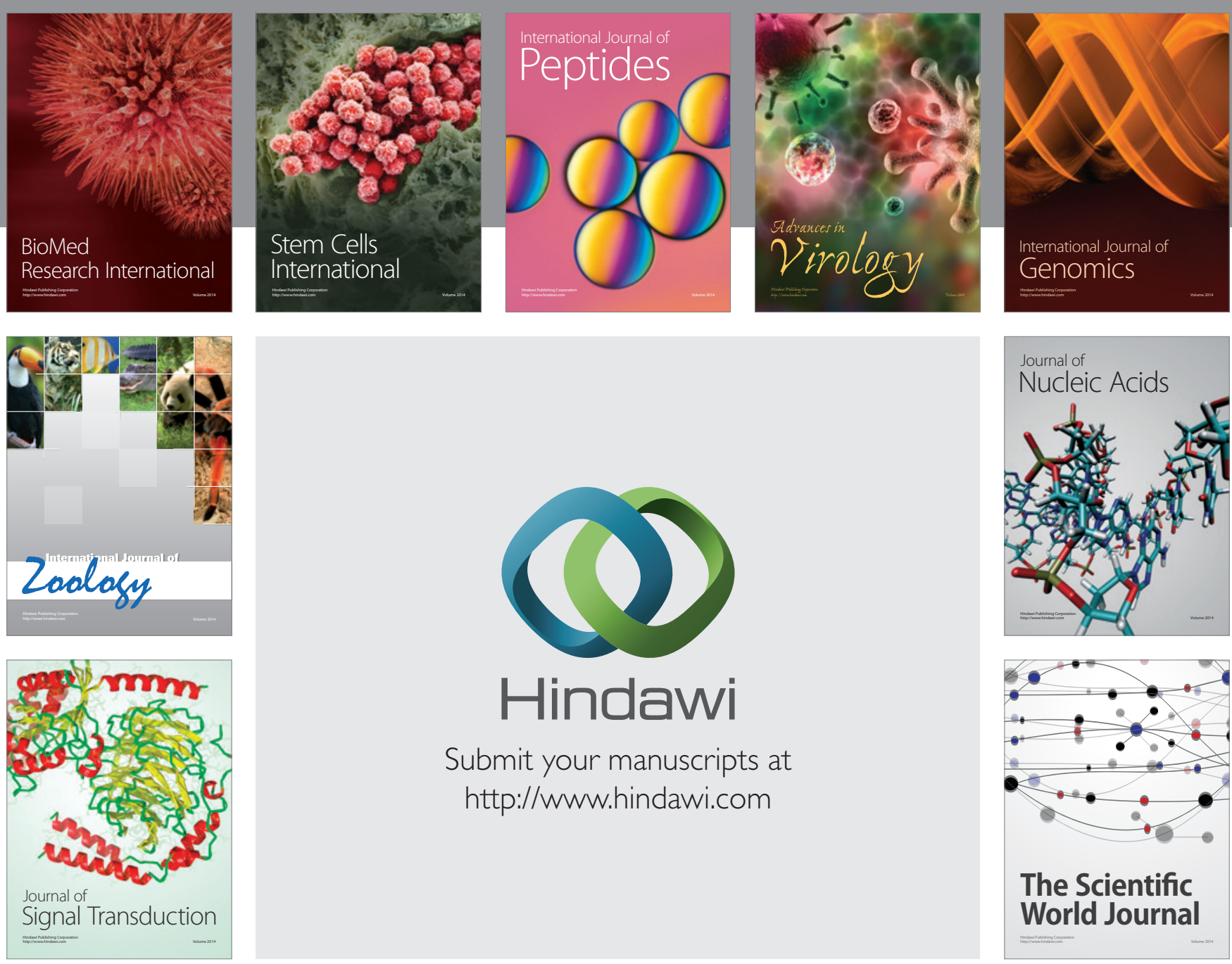

Submit your manuscripts at

http://www.hindawi.com
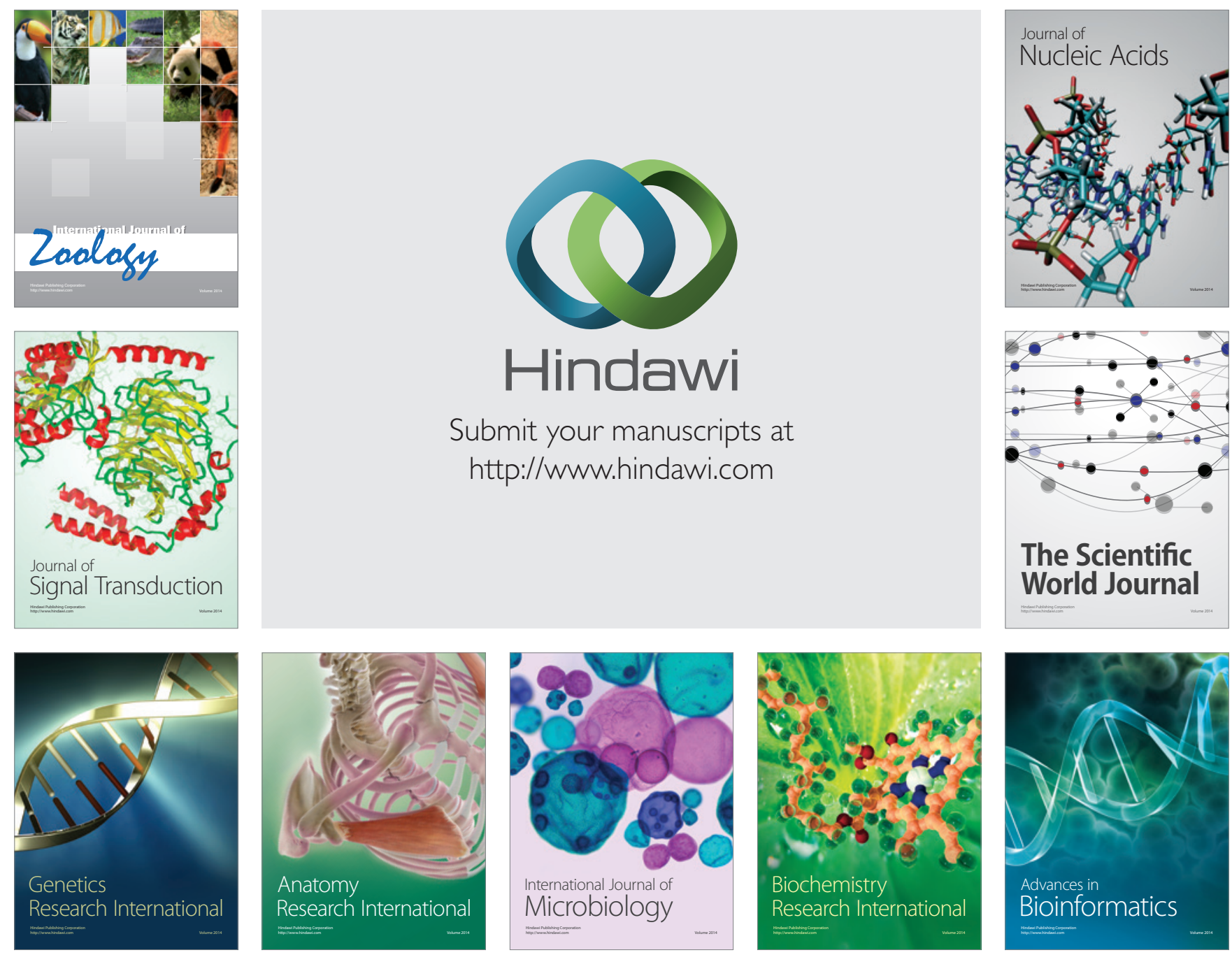

The Scientific World Journal
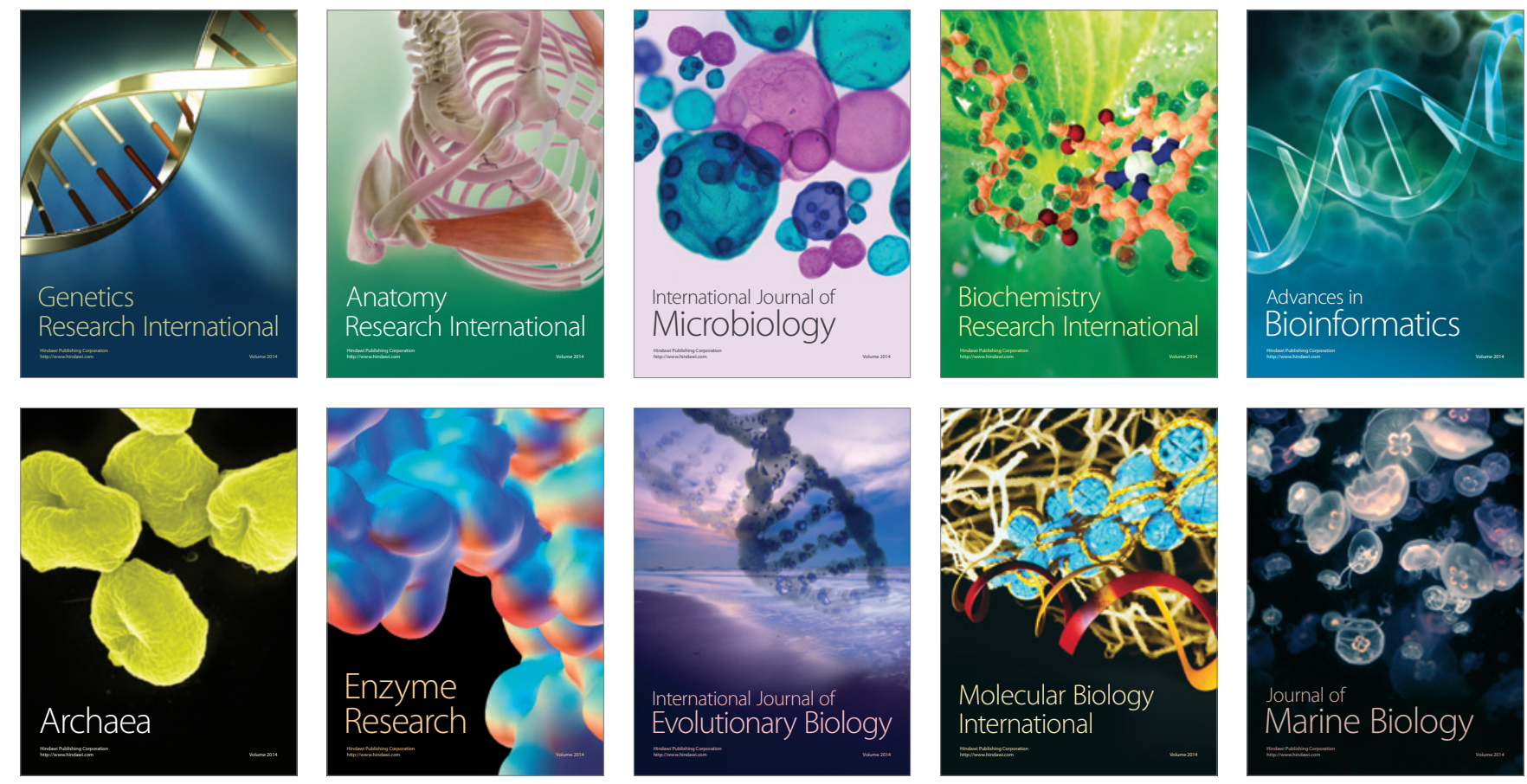